\title{
MS04-02 | Recent Advances in Cryo-Electron Tomography for In Situ Structural
}

\section{BIOLOGY}

Plitzko, Juergen (MPI of Biochemistry, Martinsried, GER)

As a consequence of the 'resolution revolution' cryo-EM has become the most versatile method for structural biology. Clearly, advances made in instrumentation, in automation and in image processing over the recent past have expanded its capabilities in a profound manner. Cryo-EM is used to determine the structure of isolated and purified macromolecular structures, i.e. ex situ but, biological functions are only rarely carried out by individual molecules. They arise from the interactions of the many molecular species found within the cells. In order to investigate these in situ, i.e. in an undisturbed cellular context, cryo-electron tomography is the method of choice. However, in tomography, several different high-end instruments are involved and many different tasks are chained into a complex, but not yet fully streamlined workflow. The fragile and delicate sample has to be prepared, information derived during these workflow steps have to be passed on and most importantly the sample has to be transferred (safely and reliably) from one system to the other. To realize the full potential of such a workflow and to apply it to many different sample materials (i.e. from cells to tissue) in a routine fashion there is an urgent need for further technology and methodology development spanning its entire range.

This lecture will present our recent work in the field of cryo-electron tomography and in situ structural biology and highlights technological developments, limitations and their opportunities and also provide a perspective for obtaining 'anatomical' detail at the molecular level from larger cells or tissues. 